New windows on massive stars: asteroseismology, interferometry, and spectropolarimetry

Proceedings IAU Symposium No. 30\%, 2014

G. Meynet, C. Georgy, J. H. Groh 8 Ph. Stee, eds.

(C) International Astronomical Union 2015 doi:10.1017/S1743921314006887

\title{
An interferometric journey around massive stars
}

\author{
Anthony Meilland and Philippe Stee \\ Laboratoire Lagrange UMR7293 OCA - CNRS - UNS - Bd de l'Observatoire, Nice, France \\ email: ame@oca.eu
}

\begin{abstract}
Since the construction in the late nineties of modern facilities such as the VLTI or CHARA, interferometry became a key technique to probe massive stars and their oftencomplex circumstellar environments. Over the last decade, the development of a new generation of beam combiners for these facilities enabled major breakthroughs in the understanding of the formation and evolution of massive stars. In this short review, we will present few of these advances concerning young stellar objects, binarity, mass loss, and stellar surfaces.
\end{abstract}

Keywords. techniques: interferometric, stars: binaries (including multiple): close, stars: circumstellar matter, stars: early-type, stars: emission-line, Be, stars: mass loss, stars: imaging

\section{Introduction}

Interferometry is the only observational technique allowing to spatially resolved structures smaller than the milliarcsecond, and that is not going to change soon. Actually, even the upcoming ELTs would have only one tenth of the current spatial resolution of CHARA (and one quarter of the VLTI).

Nevertheless, even if interferometry is the technique providing the highest spatial resolution, one interferometric observation only gives a very sparse knowledge of an object through the measurement of one or a few spatial frequencies of its Fourier transform. Thus, the more complex an object is, the more measurements are needed and, in that sense, interferometry is indeed a very time-consuming technique.

Another major issue with interferometric observations is the random wavefront perturbation stemming from the atmosphere. Such time-dependent perturbation makes it impossible to estimate the absolute interferometric phase. Unfortunately, this phase contains most of the information on a object as shown in Fig. 1 where either the phase or the modulus of Fourier transform is replaced by a random distribution. Despite this impossibility to directly measure the phase, partial information can be retrieve using a linear combination of the measured phases on three telescopes, called closure phase, which cancels the atmospheric contributions.

The recent increase in number of telescopes of the interferometric arrays and the improve capability of the new instruments that can simultaneously combine the light from more telescopes, with a current record of six for the MIRC instrument installed on CHARA, partly solved these issues. It allows to simultaneously measure more visibilities (up to 15) and closure phases (up to 20). With such an improvement compared to previous facilities or instruments working with two or three telescopes, better and faster sampling of the object Fourier transform can be obtained, and finally, image reconstruction techniques can be used efficiently.

In the following we will present a short review of some of the last-decade major results obtained using optical interferometry on the formation (Sect. 2), the binarity (Sect. 3), the circumstellar environment (Sect. 4), and the surface (Sect. 5) of massive stars. 


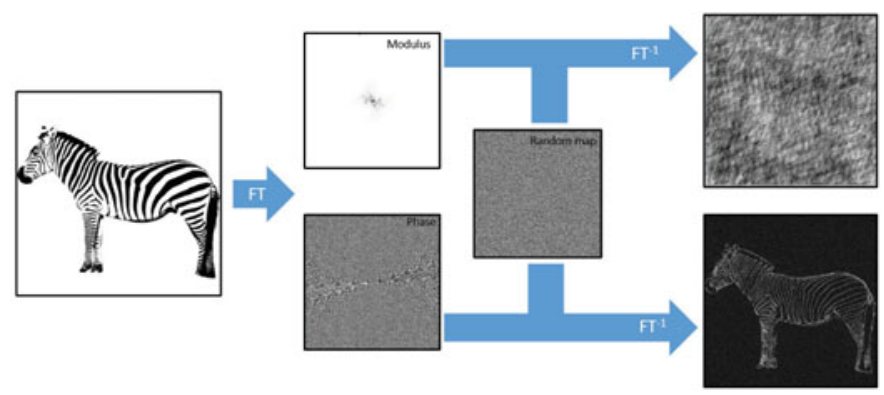

Figure 1. Example illustrating that most of the information on an object geometry is contained in the phase part of its Fourier transform and not in its modulus.

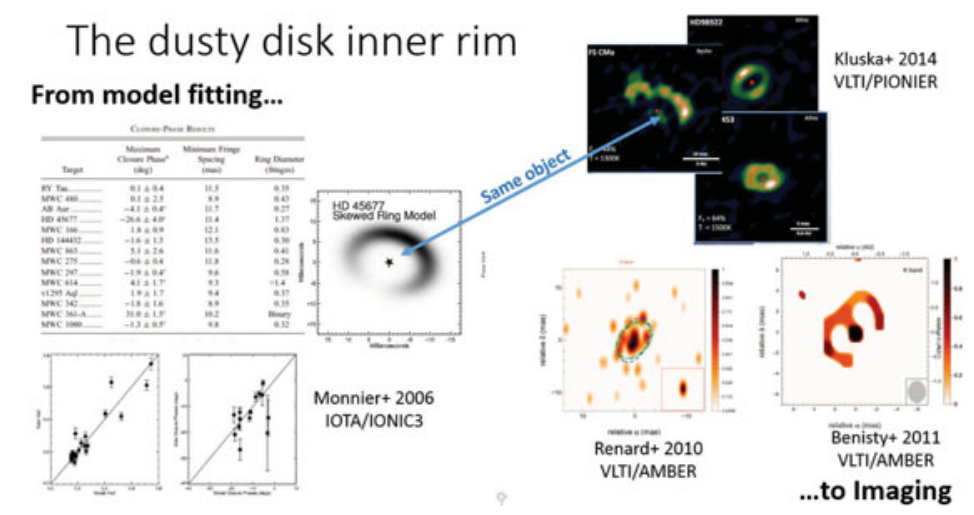

Figure 2. The inner rim of dusty disk around Herbig Ae/Be stars seen by optical interferometry. From Monnier et al. (2006), Renard et al. (2010), Benisty et al. (2011), and Kluska et al. (2014).

\section{The formation of massive stars}

The gain in sensibility of modern optical interferometers made it possible to observe the circumstellar environment of young stellar objects (YSO). Many studies were conducted in the near-infrared to constrain the dust sublimation radius and in the mid-infrared to probe colder material further from the central star. From PTI, IOTA, and Keck aperture masking measurements, Monnier \& Millan-Gabet (2002) presented the first statistical study of the near-IR extension of YSO. This work was then completed by Keck-I measurements (Monnier et al. 2005) and presented in a more complete review in Dullemond \& Monnier (2010). The relation they derived was compatible with simple dusty disk models with an inner rim at an expected dust sublimation temperature.

However, to discriminate between different disk models, a more detailed view of the dusty disk inner rim was needed. For instance, the measure of inner-rim skewness, that can only be done using closure phases, can help to put constraints on the inner-rim height and the disk opacity. First measurements of this skewness was done by Monnier et al. (2006) using IOTA. More recent facilities such as VLTI/AMBER and VLTI/PIONIER were used to obtain images of the inner-rim (Renard et al. 2010, Benisty et al. 2011, and Kluska et al. 2014). These results are summarized in Fig. 2.

Similar studies were conducted in the mid-infrared, mainly with the VLTI/MIDI (Leinert et al. 2004, Preibisch et al. 2006 ,di Folco et al. 2009), to probe the larger scale environment and constrain the disk density distribution, flaring, temperature law, and 
chemistry thanks to MIDI high-enough spectral resolution, that allows to resolve the large PAH and silicate spectral features.

On the other hand, a higher spectral resolution was needed to resolve the hydrogen emission lines and study the geometry and kinematics of the circumstellar gas. Such studies were made possible with the VLTI/AMBER whose resolution is $\mathrm{R}=1500$ in MR mode and $\mathrm{R}=12000$ in HR mode. The first study was done by Malbet et al. (2007) on MWC 297. They showed that, for this object, the $\operatorname{Br} \gamma$ emission was coming from a region larger than the dusty-disk inner rim and that it was expanding, ruling out the possibility of an emission coming from an inner gaseous disk. This object was observed again by Weigelt et al. (2011) with a higher spectral resolution and they managed to fully model their observations with a disk-wind model. For an other object, HD 104237, Tatulli et al. (2007) found that the extension of the gaseous emission was of the order of the dusty disk inner rim.

The first small survey on the gaseous emission of Herbig stars was published in Kraus et al. (2008). They have shown that the gaseous emission can stem from various regions in the circumstellar environment. Of the five stars in their survey, two had emission originating from compact regions compatible with the inner disk. For the three others, the emission was at least as extended as the disk inner rim, favoring the hypotheses of a disk-wind or a very extended stellar wind.

Finally, the VEGA instrument, working in the visible and installed in 2008 on the CHARA array, made it possible to study the gas in the $\mathrm{H} \alpha$ emission line. Two objects were studied : AB Aur by Rousselet-Perraut et al. (2010) and HD 200775 by Benisty et al. (2013). They both show an extended gaseous emission compatible with a wind model, but highly flattened in the case of AB Aur.

All these previously-cited studies concern stars up to about $10 M_{\odot}$. The formation processes of the more massive stars are still high debated. Actually, two main scenarios are competing to explain their formation. One is a scaled-up version of Herbig stars scenario, an accretion disk phase followed by a blow-up of the remaining material by the stellar wind. The second one called "competitive accretion", involves a high stellar density (cluster cores), which attracts and forces accretion onto the most massive stars. In this scenario all massive stars should belong to multiple systems. The first very massive young stars observed with the VLTI/AMBER (Kraus et al. 2010) showed no clue of binarity and a disk-like structure compatible with the first scenario (see Fig. 3). However, such result need to be confirmed on a larger sample of massive young stellar object, and the binary fraction of massive stars should also be measured.
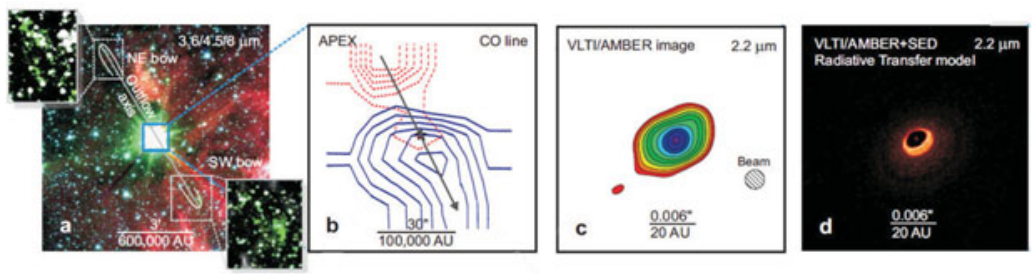

Figure 3. Zoom in on the massive star IRAS 13481-6124 (Kraus et al. 2010). From left to right: Spitzer and APEX images showing a bipolar outflow, VLTI/AMBER image and corresponding best-fit radiative model showing a disk like structure perpendicular to the outflow. 

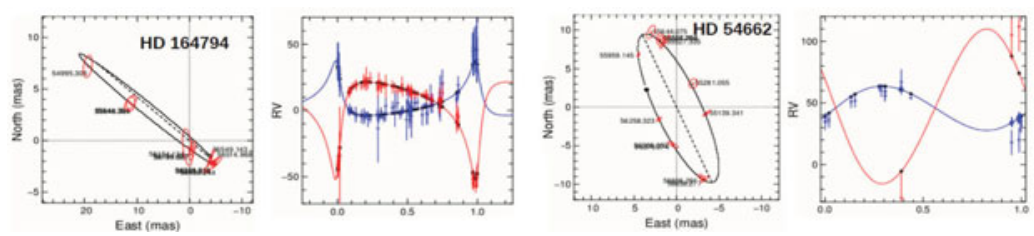

Figure 4. Examples of two massive stars 3D-orbit constrained using interferometric and radial velocity measurements. From yet unpublished VLTI/PIONIER data.

\section{The multiplicity of massive stars}

Optical interferometry is also a suitable technique for studying stellar multiplicity, especially when it comes to systems with separation of the order of the milliarcsecond. For instance, in an attempt to determine the binary ratio of massive stars, a joint program using the VLTI/PIONIER for small separation (1 to 45 mas) and the VLT/NACO for larger separation (30 mas to 8") was initiated. 173 southern O stars were observed during this Southern Massive stArs at High Angular Resolution (SMASH+) program. The results of this survey, published in Le Bouquin (2014), showed a very high binary fraction of the sample, 0.86 , with an average number of companions of $1.83 \pm 0.32$. They conclude that almost all massive stars are in multiple systems.

Optical interferometry can also be used to determine the visual orbit of binary systems (i.e. projected onto the sky-plane). Combined with spectroscopic measurements of radial velocity, the full three-dimensional orbit of the system can be constrained. This allows to derive the total mass of the system and of the masses of its components with a precision down to a few percents. Many works has been done on massive binary systems, for instance: Sana et al. (2013) on the triple O system HD 150136 using the VLTI/PIONIER, Kraus et al. (2009) on the binary YSO $\theta^{1}$ Ori C with the VLTI/AMBER, or Monnier et al. (2011) of the Wolf-Rayet binary WR104 using CHARA/CLASSIC and IOTA/IONIC3. Figure 4 presents the case of two massive stars recently observed with the VLTI/PIONIER.

During their evolution, stars in binary systems can interact with each other through their circumstellar or circumbinary environment. Such interactions, that can be once again well constrained using optical interferometry, are mainly due to gravitational effects or particle collisions (mainly in stellar winds).

The Be-binary system $\beta$ Lyr is a good example of what can be done on interacting systems using interferometry . This semidetached binary is composed of two B-type stars, a primary supergiant filling its Roche lobe, and a secondary surrounded by an accretion disk fed by the primary material leaking in through the system's first Lagrangian point. Near-infrared reconstructed-images during a complete orbital period were obtained by Zhao et al. (2008) using the CHARA/MIRC combiner (see Fig. 5). On these images two components are seen, one unresolved, the primary, and one elongated, the secondary surrounded by the accretion disk. To go further in this study, and disentangle the stellar and circumstellar emission, the system was also observed in $\mathrm{H} \alpha$ with the CHARA/VEGA instrument (Bonneau et al. 2011). The authors found that the emission was coming from a structure larger than the binary separation, most probably a circumbinary disk with possible contributions from polar jets.

Another interacting binary system discovered and studied using interferometry is the yellow hypergiant HR 5171A. Chesneau et al. (2014) showed that this star, one of the largest known star, i.e. $R \sim 1300 R_{\odot}$, is in contact with a previously unknown companion, and that the system is probably in a common envelope phase.

Due to a lack of sensibility, only a few studies of wind collisions were conducted using interferometry. The main results were obtained with the Keck aperture masking 

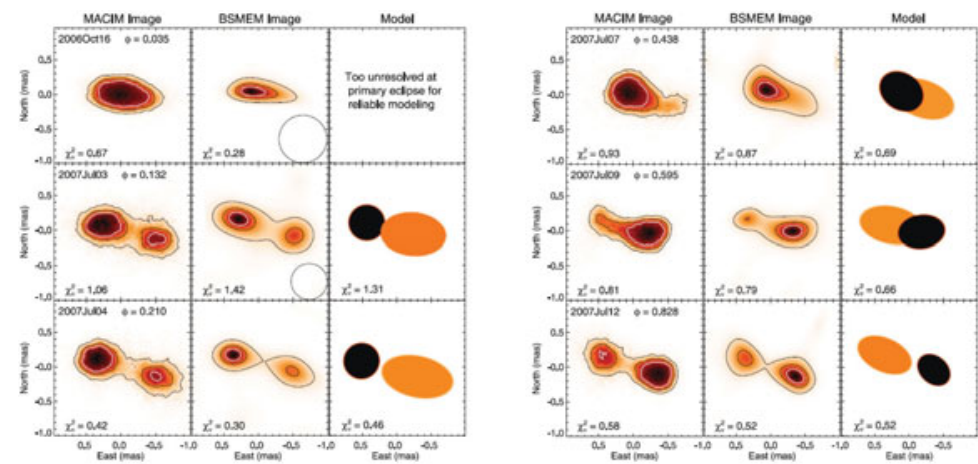

Figure 5. The Be-binary system $\beta$ Lyr as seen by CHARA/MIRC during one full orbital period. From Zhao et al. (2008).

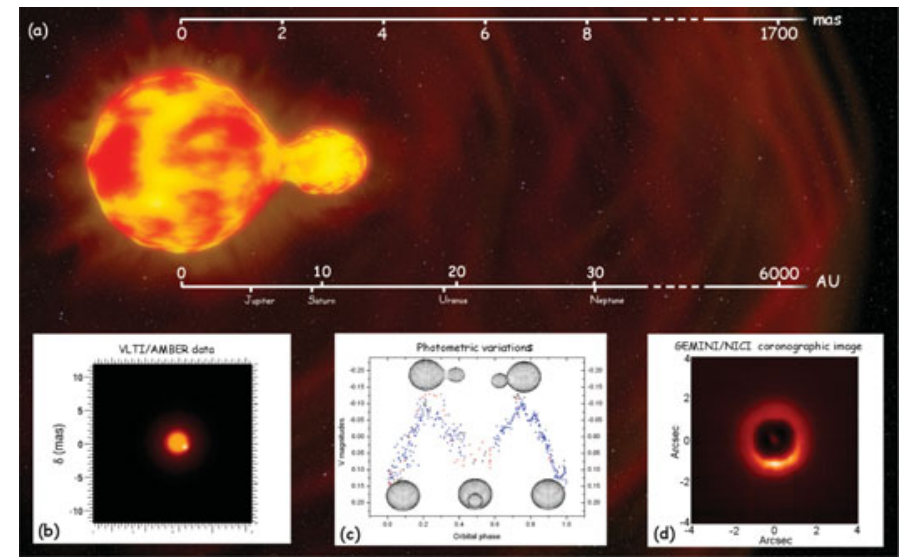

Figure 6. VLTI/AMBER observations and long term photometry revealed the binary nature and common envelope phase of the yellow hypergiant HR 5171A. From Chesneau et al. (2014).

experiment (Tuthill et al. 1999, Harries et al. 2004 and Monnier et al. 2007a) on imaging of dust around Wolf-Rayet stars. They found out that the dust was located in a pinwheel nebulae, i.e. a spiral-like pattern tracing the orbital motion of the wind-collision layer. The first Wolf-Rayet stars observed with long-baseline optical interferometry were $\gamma^{2}$ Vel (Millour et al. 2007) and WR 118 (Millour et al. 2009b). In both papers, the authors manage to resolve the system, and in that second one, they found closure phase signal compatible with a pinwheel model.

\section{Mass loss and circumstellar environments}

As a natural tracer of mass loss, the study of massive stars circumstellar environment is crucial to improve their evolution models. To characterize not only the total mass loss but its possible anisotropy, one has to put some constraints on the geometry and kinematics of the close surrounding of these massive stars. Interferometry and in particular spectro-interferometry are indeed powerful techniques to probe these environments whose extensions are usually of the order of few millarscseconds in the visible and near-IR and tens of milliarseconds in the mid-IR.

Chesneau et al. (2010) used the CHARA/VEGA instrument to study the wind of two blue supergiants: Deneb and Rigel. He managed to model these high-spectral resolution 

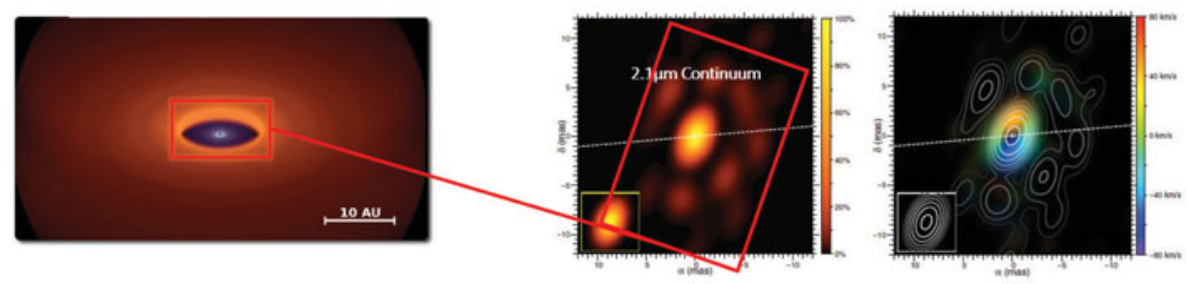

Figure 7. The A[e] supergiant seen by the VLTI instruments MIDI (left) and AMBER, in the $2.1 \mu \mathrm{m}$ continuum (center) and the Br $\gamma$ line (right). From Meilland et al. (2010) and Millour et al. (2011).

$(\mathrm{R}=30000)$ observations centered on $\mathrm{H} \alpha$ using the CMFGEN radiative transfer code. The authors also found a strong asymmetry in Deneb wind.

Using VLTI/AMBER and VLTI/VINCI measurements Groh et al. (2010) probed the heart of $\eta$ Car circumstellar environment. They studied possible effects from fast-rotation of the primary and wind collision to explain the break of symmetry of the mass loss and the formation of the Homonculus nebula around these very massive objects. The interferometric study of $\eta$ Car is still going on and new results are presented further in this book.

Actually, the mass-loss break of symmetry, its origin and its consequences are major issues in the understanding of stellar evolution. Binarity and rotation are the most common physical phenomena that can cause departure from a spherically symmetric mass loss, affecting the loss of angular momentum of the star itself.

Among the objects concerned by this break of symmetry are the stars showing the $\mathrm{B}[\mathrm{e}]$ phenomenon, i.e. presence of forbidden lines produced in a diluted and highly illuminated medium and of strong IR-excess due to the presence of dust produced in a dense and cool medium. Both rotation through the bi-stable model and binarity were proposed to explain the formation of an equatorial structure dense enough to allow dust formation in their circumstellar environment.

These $\mathrm{B}[\mathrm{e}]$ stars were perfect targets for the VLTI instruments MIDI and AMBER and the brightest of them were studied in the last decade : CPD $-57^{\circ} 2874$ (Domiciano de Souza et al. 2007), Hen 3-1191 (Lachaume et al. 2007), HD 87643 (Millour et al. 2009a), HD 62623 (Meilland et al. 2010, Millour et al. 2011), HD 50138 (Borges Fernandes et al. 2011), MWC 300 (Wang et al. 2012), HD 327083 (Wheelwright et al. 2012), V921 Sco (Kreplin et al. 2012), and HD 85567 (Wheelwright et al. 2013). Most of these study modeled either with simple geometric model or radiative transfer code such as MC3D, conclude that the dust was located in a circumstellar disk and that the gaseous emission was coming from structures smaller than the dusty-disk inner rim.

In Fig. 7 we present the results obtained on the most extensively studied object with both MIDI and AMBER : the A[e] supergiant HD 62623. For this object, the authors found that both the gas and dust formed a stratified disk in Keplerian rotation, and that the stellar velocity was too small for the rotation to explain the break of symmetry of the environment but too high for a supergiant. They concluded that this star has probably gone through a spin-up phase due to interaction with a spectroscopically detected companion. However, the detailed mechanisms for the disk formation remain unknown.

Classical Be stars were already favored targets for interferometers in the nineties. However, these studies were quite limited by the data lack of accuracy and the small number of telescopes of the interferometric arrays. In the last decade, many objects were observed with modern facilities, and data were analyzed using either simple geometric or kinematic models (Tycner et al. 2004, Tycner et al. 2006, Meilland et al. 2008, Delaa et al. 2011, 

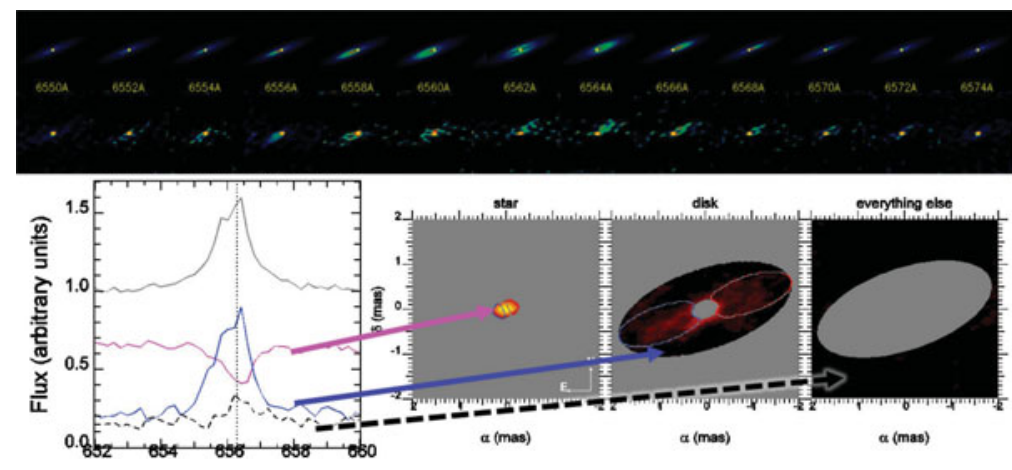

Figure 8. Narrow-spectral band images in the $\mathrm{H} \alpha$ line (top) and extracted spectrum for the star and disk (bottom) for the Be-shell star $\phi$ Per. From CHARA/VEGA measurement to be published in Mourard et al. (2014).

Stee et al. 2012, Kraus et al. 2012) or radiative transfer codes such as SIMECA (Meilland et al. 2007a, Meilland et al. 2007b), BEDSIK (Tycner et al. 2011), or HDUST (Štefl et al. 2009, Carciofi et al. 2009). In all cases the authors found that the circumstellar emission stems from a thin equatorial disk. The more detailed studies, using spectral resolution allowed to probe the kinematics and show that disks were dominated by rotation, probably close to the Keplerian one. The disk density law was also constrained, showing that the observations were compatible with the viscous excretion disk model.

Two Be binary stars were extensively studied to understand the effect of binarity on the Be phenomenon : $\delta$ Sco (Tycner et al. 2011, Meilland et al. 2011, Meilland et al. 2013) and Achernar (Kervella \& Domiciano de Souza 2006, Kanaan et al. 2008, Kervella et al. 2009). Finally, some statistical studies of Be stars environments were conducted to constrain the general properties of their disks (Gies et al. 2007, Meilland et al. 2009, Touhami et al. 2013) and also to derive a mean rotational rate of Be stars showing that they were very close to their critical rotation (Meilland et al. 2012).

Fig. 8 shows an example of the possibility of Be stars observations with modern spectrointerferometric instruments. In this forthcoming paper, Mourard et al. (2014) manage to reconstruct narrow-spectral band images in the $\mathrm{H} \alpha$ line probing the disk geometry and kinematics of this Be-shell star. As shown in the figure, the technique is similar to integral-field spectroscopy but with a forty-times higher spatial resolution.

\section{From diameters measurements to stellar imaging}

Stellar radius is one of the main input parameters of stellar interior models. It is often used to derive the star mass, age and other physical parameters. With no direct angular measurements, the radius is generally inferred from photometry but with some important biases and poor accuracy. Modern interferometers allow to obtain accurate angular diameters, and using Hipparcos parallaxes, to derive more accurate linear radii.

Accurate angular diameters are also needed to calibrate the surface-brightness relation for some distance estimators such Cepheids and eclipsing binaries. Up to recently, such relation was poorly constrained for early type stars. Recently, significant improvements (see Fig. 9) were obtained using the two CHARA visible beam-combiners, PAVO (Maestro et al. 2013) and VEGA (Challouf et al. 2014).

Actually, the first star measured with interferometry almost a hundred years ago, the red supergiant Betelgeuse, is still being observed with modern facilities. Haubois et al. (2009) obtained the first reconstructed image of its stellar surface. He managed 

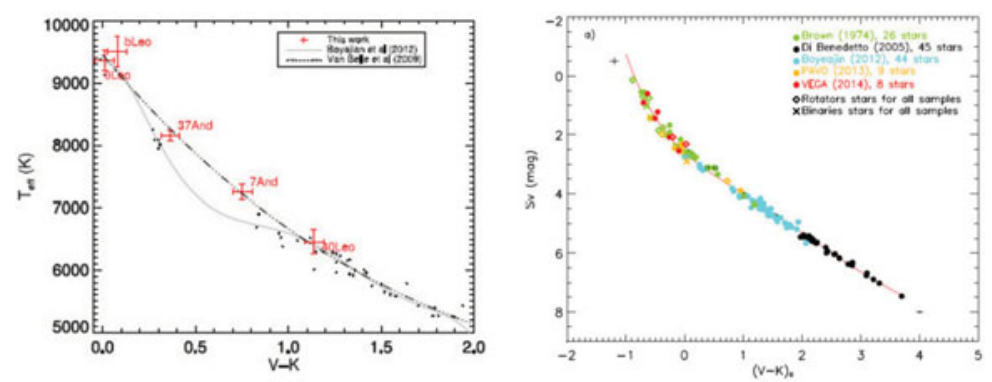

Figure 9. Surface brightness relation for early type stars determined with CHARA/PAVO (Maestro et al. 2013, left) and CHARA/VEGA (Challouf et al. 2014,right).
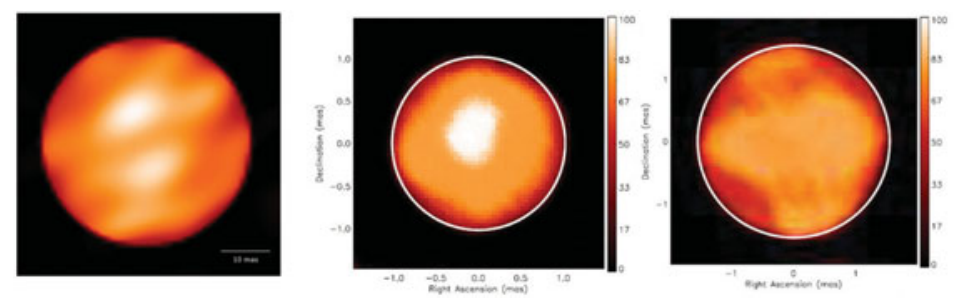

Figure 10. Reconstructed images of red supergiants: Betelgeuse (left), RS Per (center) and T Per (right). From Haubois et al. (2009) and Baron et al. (2014).

to detect giant "spots" on the surface that are compatible with convection cells (see Fig. 10). Two other red supergiants were imaged by (Baron et al. 2014) in the K-band continuum with CHARA/MIRC whereas the yellow supergiant Canopus was resolved by the VLTI/AMBER (Domiciano de Souza et al. 2012).

On the other hand, using the VLTI/AMBER spectro-interferometric capability, Ohnaka et al. (2009) resolved the $2.3 \mu \mathrm{m}$ CO bands of Betelgeuse. These observations allowed to study the geometry and kinematics, showing evidence of asymmetry and convection in a $1.3 R_{\star}$ extended atmosphere. New observations published in Ohnaka et al. (2011) showed significant changes in the kinematics and geometry. Similar results were found for an other red supergiant, i.e. Antares (Ohnaka et al. 2013).

Stellar surfaces of five intermediate-mass fast-rotators were imaged in the $\mathrm{K}$ band using the CHARA/MIRC instrument (Monnier et al. 2007b, Zhao et al. 2009, Che et al. 2011). The reconstructed images, presented in Fig. 11, allowed to constrain the photosphere flattening and gravity darkening induced by the Von Zeipel effect. Actually, the authors found that their observations are better fitted by a $\beta$ exponent of 0.22 , i.e. slightly different from the 0.25 value derived in Von Zeipel work for radiative stars. Finally, using the ESTER model for interior of fast rotating stars, Espinosa Lara \& Rieutord (2011) found values compatible to the one measured by MIRC.

However, Delaa et al. (2013) showed that the measured value of the $\beta$ parameter can be biased in case of a latitudinal differential rotation. Such degeneracy on the gravity darkening measurement could be removed using spectro-interferometry in photospheric lines as show in Fig. 12.

Finally, the differential phase measurements can also be used to measure the stellar diameters of unresolved fast-rotators as shown by Domiciano de Souza et al. (2012) using VLTI/AMBER high-resolution measurements of the close-to-critical rotator Achernar. Using the same technique Hadjara et al. (2014) even managed to derive the radius for slower rotators. 


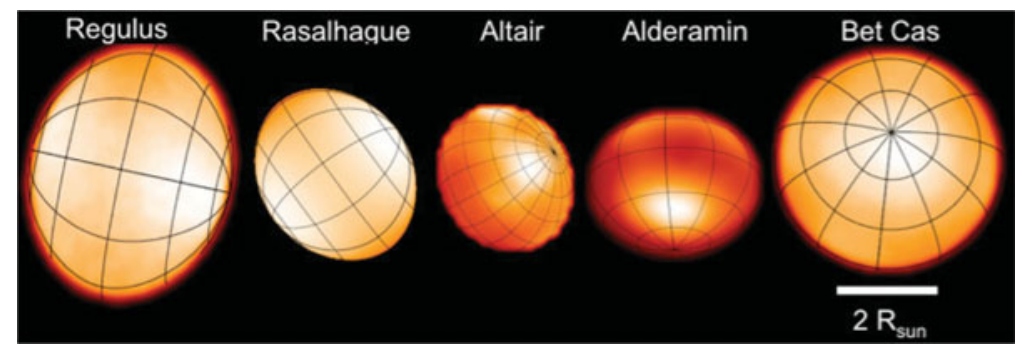

Figure 11. Fast rotators as seen by CHARA/MIRC. From Monnier et al. (2007b), Zhao et al. (2009), and Che et al. (2011).

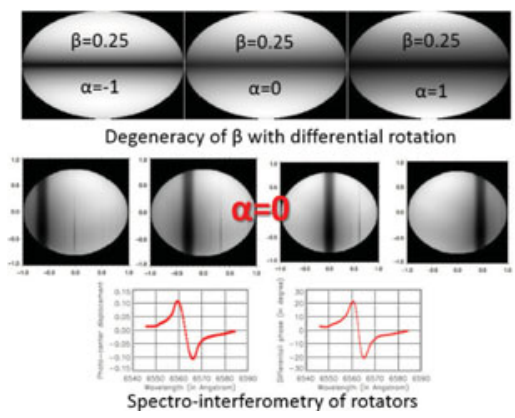

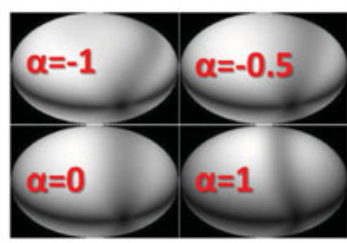

Effects of latitudinal differential rotation On narrow band images

Figure 12. Stellar rotation probed by spectro-interferometry. Top left: Intensity map in the continuum showing the degeneracy of the $\beta$ parameters for different values of the latitudinal-differential rotation parameter $\alpha$. Bottom left: Narrow band images at different wavelengths through a photopheric line for $\alpha=0$ and corresponding differential phase signal. Narrow band images at one wavelength for different values of the $\alpha$ parameter. From Delaa et al. (2013).

\section{References}

Baron, F., Monnier, J. D., Kiss, L. L., et al. 2014, ApJ 785, 46

Benisty, M., Perraut, K., Mourard, D., et al. 2013, A\&SA 555, A113

Benisty, M., Renard, S., Natta, A., et al. 2011, A\& $A$ 531, A84

Bonneau, D., Chesneau, O., Mourard, D., et al. 2011, A\&SA 532, A148

Borges Fernandes, M., Meilland, A., Bendjoya, P., et al. 2011, A $\mathscr{E} A$ 528, A20

Carciofi, A. C., Okazaki, A. T., Le Bouquin, J.-B., et al. 2009, A $\& A$ 504, 915

Challouf, M., Nardetto, N., Mourard, D., et al. 2014, Submitted to A\&A

Che, X., Monnier, J. D., Zhao, M., et al. 2011, ApJ 732, 68

Chesneau, O., Dessart, L., Mourard, D., et al. 2010, A\&A 521, A5

Chesneau, O., Meilland, A., Chapellier, E., et al. 2014, A\&SA 563, A71

Delaa, O., Stee, P., Meilland, A., et al. 2011, A\& $A$ 529, A87

Delaa, O., Zorec, J., Domiciano de Souza, A., et al. 2013, A\&SA 555, A100

di Folco, E., Dutrey, A., Chesneau, O., et al. 2009, A\&A 500, 1065

Domiciano de Souza, A., Driebe, T., Chesneau, O., et al. 2007, A\&\&A 464, 81

Domiciano de Souza, A., Hadjara, M., Vakili, F., et al. 2012, A\&\&A 545, A130

Dullemond, C. P. \& Monnier, J. D. 2010, ARA $\& A$ 48, 205

Espinosa Lara, F. \& Rieutord, M. 2011, A $\& A$ 533, A43

Gies, D. R., Bagnuolo, Jr., W. G., Baines, E. K., et al. 2007, ApJ 654, 527

Groh, J. H., Madura, T. I., Owocki, S. P., Hillier, D. J., \& Weigelt, G. 2010, ApJ (Letters) 716, L223

Hadjara, M., Domiciano de Souza, A., Vakili, F., et al. 2014, Submitted to A\&\&A

Harries, T. J., Monnier, J. D., Symington, N. H., \& Kurosawa, R. 2004, MNRAS 350, 565

Haubois, X., Perrin, G., Lacour, S., et al. 2009, A\&A 508, 923

Kanaan, S., Meilland, A., Stee, P., et al. 2008, A\&A 486, 785 
Kervella, P. \& Domiciano de Souza, A. 2006, A 6 A 453, 1059

Kervella, P., Domiciano de Souza, A., Kanaan, S., et al. 2009, A\&A 493, L53

Kluska, J., Malbet, F., Berger, J.-P., et al. 2014, in M. Booth, B. C. Matthews, \& J. R. Graham (eds.), IAU Symposium, Vol. 299 of IAU Symposium, pp 117-118

Kraus, S., Hofmann, K.-H., Benisty, M., et al. 2008, A\&SA 489, 1157

Kraus, S., Hofmann, K.-H., Menten, K. M., et al. 2010, Nature 466, 339

Kraus, S., Monnier, J. D., Che, X., et al. 2012, ApJ 744, 19

Kraus, S., Weigelt, G., Balega, Y. Y., et al. 2009, A\&A 497, 195

Kreplin, A., Kraus, S., Hofmann, K.-H., et al. 2012, A\& A 537, A103

Lachaume, R., Preibisch, T., Driebe, T., \& Weigelt, G. 2007, A\&A 469, 587

Le Bouquin, J.-B. 2014, Submitted to A\&A

Leinert, C., van Boekel, R., Waters, L. B. F. M., et al. 2004, A $\& A 423,537$

Maestro, V., Che, X., Huber, D., et al. 2013, MNRAS 434, 1321

Malbet, F., Benisty, M., de Wit, W.-J., et al. 2007, A\&SA 464, 43

Meilland, A., Delaa, O., Stee, P., et al. 2011, A\&A 532, A80

Meilland, A., Kanaan, S., Borges Fernandes, M., et al. 2010, A $\& A$ 512, A73

Meilland, A., Millour, F., Kanaan, S., et al. 2012, A\&A 538, A110

Meilland, A., Millour, F., Stee, P., et al. 2007a, A\&SA 464, 73

Meilland, A., Millour, F., Stee, P., et al. 2008, A\&A 488, L67

Meilland, A., Stee, P., Chesneau, O., \& Jones, C. 2009, A $\& A$ A 505, 687

Meilland, A., Stee, P., Spang, A., et al. 2013, A $6 A$ 550, L5

Meilland, A., Stee, P., Vannier, M., et al. 2007b, A\& 4 464, 59

Millour, F., Chesneau, O., Borges Fernandes, M., et al. 2009a, A\&A 507, 317

Millour, F., Driebe, T., Chesneau, O., et al. 2009b, A\&A 506, L49

Millour, F., Meilland, A., Chesneau, O., et al. 2011, A\&A 526, A107

Millour, F., Petrov, R. G., Chesneau, O., et al. 2007, A\&A 464, 107

Monnier, J., Millan-Gabet, R., \& Billmeier, R. 2005, ApJ 624, 832

Monnier, J. D., Berger, J.-P., Millan-Gabet, R., et al. 2006, ApJ 647, 444

Monnier, J. D. \& Millan-Gabet, R. 2002, ApJ 579, 694

Monnier, J. D., Tuthill, P. G., Danchi, W. C., Murphy, N., \& Harries, T. J. 2007a, ApJ 655, 1033

Monnier, J. D., Zhao, M., Pedretti, E., et al. 2011, ApJ (Letters) 742, L1

Monnier, J. D., Zhao, M., Pedretti, E., et al. 2007b, Science 317, 342

Mourard, D., Monnier, J., Che, X., Meilland, A., \& Millour, F. 2014, Submitted to A\&A

Ohnaka, K., Hofmann, K.-H., Benisty, M., et al. 2009, A\&GA 503, 183

Ohnaka, K., Hofmann, K.-H., Schertl, D., et al. 2013, A $\& A$ 555, A24

Ohnaka, K., Weigelt, G., Millour, F., et al. 2011, A\&A 529, A163

Preibisch, T., Kraus, S., Driebe, T., van Boekel, R., \& Weigelt, G. 2006, A $\& A$ 458, 235

Renard, S., Malbet, F., Benisty, M., Thiébaut, E., \& Berger, J.-P. 2010, A\& $A$ 519, A26

Rousselet-Perraut, K., Benisty, M., Mourard, D., et al. 2010, A\&A 516, L1

Sana, H., Le Bouquin, J.-B., Mahy, L., et al. 2013, A\&A 553, A131

Stee, P., Delaa, O., Monnier, J. D., et al. 2012, A\&A 545, A59

Tatulli, E., Isella, A., Natta, A., et al. 2007, A\& $A$ 464, 55

Touhami, Y., Gies, D. R., Schaefer, G. H., et al. 2013, ApJ 768, 128

Tuthill, P. G., Monnier, J. D., \& Danchi, W. C. 1999, Nature 398, 487

Tycner, C., Ames, A., Zavala, R. T., et al. 2011, ApJ (Letters) 729, L5

Tycner, C., Gilbreath, G. C., Zavala, R. T., et al. 2006, AJ 131, 2710

Tycner, C., Hajian, A. R., Armstrong, J. T., et al. 2004, AJ 127, 1194

Štefl, S., Rivinius, T., Carciofi, A. C., et al. 2009, A\& $A$ 504, 929

Wang, Y., Weigelt, G., Kreplin, A., et al. 2012, A\& A 545, L10

Weigelt, G., Grinin, V. P., Groh, J. H., et al. 2011, A\&A 4 527, A103

Wheelwright, H. E., de Wit, W. J., Oudmaijer, R. D., \& Vink, J. S. 2012, A\&\&A 538, A6

Wheelwright, H. E., Weigelt, G., Caratti o Garatti, A., \& Garcia Lopez, R. 2013, A\&A 558, A116 
Zhao, M., Gies, D., Monnier, J. D., et al. 2008, ApJ (Letters) 684, L95

Zhao, M., Monnier, J. D., Pedretti, E., et al. 2009, ApJ 701, 209

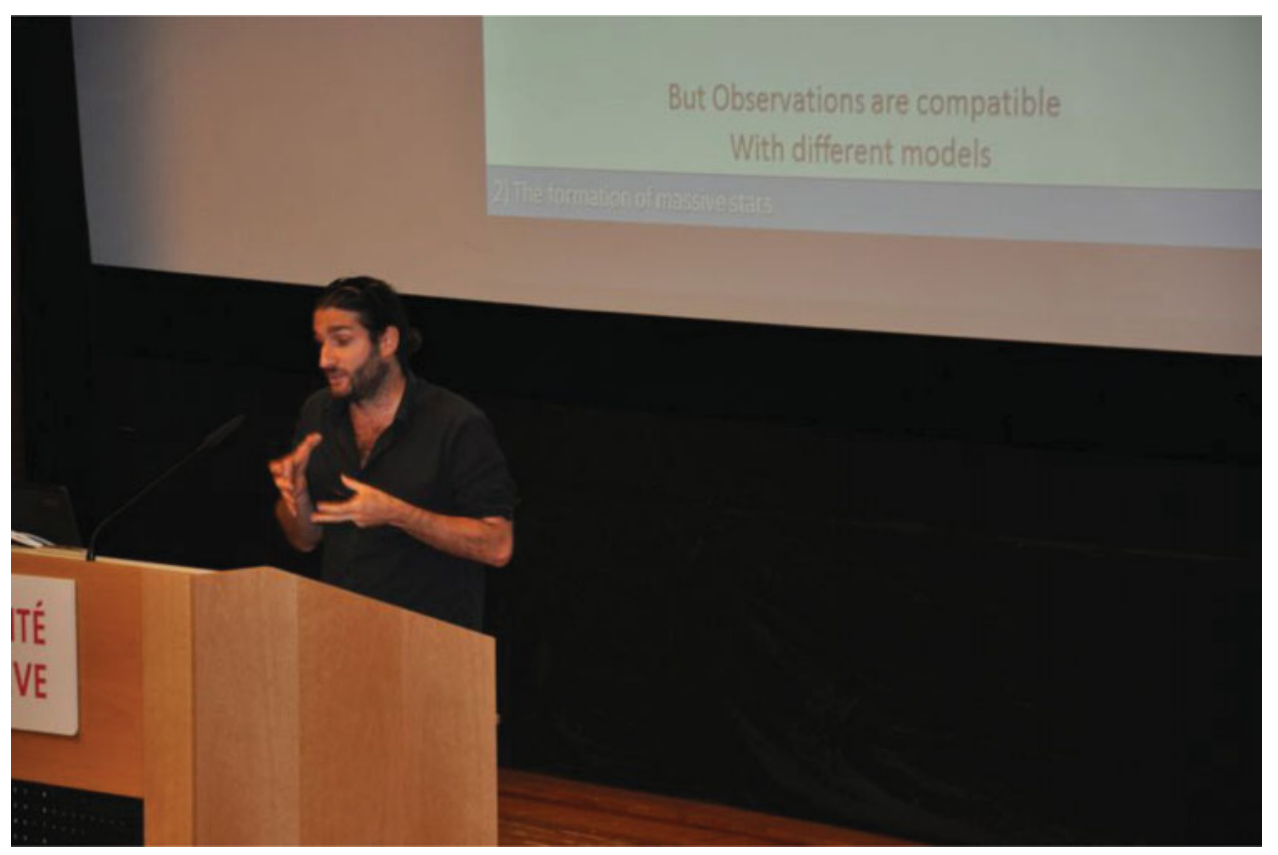

Anthony Meilland 